Check for updates

Cite this: Phys. Chem. Chem. Phys., 2018, 20, 27342

Received 10th August 2018, Accepted 8th October 2018

DOI: $10.1039 / c 8 c p 05107 k$

rsc.li/pccp

\title{
Why is the change of the Johari-Goldstein $\beta$-relaxation time by densification in ultrastable glass minor? $\dagger$
}

\author{
K. L. Ngai, ${ }^{\star a}$ Marian Paluch ${ }^{\text {bc }}$ and Cristian Rodriguez-Tinoco (D) $\star^{b c}$
}

\begin{abstract}
Ultrastable glasses (USG) formed by vapor deposition are considerably denser. The onset temperature of devitrification, $T_{\text {on }}$ is significantly higher than $T_{\text {on }}$ or $T_{\mathrm{g}}$ of ordinary glass (OG) formed by cooling, which implies an increase of the structural $\alpha$-relaxation time by many orders of magnitude in USG compared to that in $O G$ at the same temperature. However, for a special type of secondary relaxation having properties strongly connected to those of the $\alpha$-relaxation, called the Johari-Goldstein $\beta$-relaxation, its relaxation time in USG is about an order of magnitude slower than that in OG and it has nearly the same activation energy, $E_{\beta}$. The much smaller change in $\tau_{\beta}$ and practically no change in $E_{\beta}$ by densification in USG are in stark contrast to the behavior of the $\alpha$-relaxation. This cannot be explained by asserting that the Johari-Goldstein (JG) $\beta$-relaxation is insensitive to densification in USG, since the JG $\beta$-relaxation strength is significantly reduced in USG to such a level that it would require several thousands of years of aging for an OG to reach the same state, and therefore the JG $\beta$-relaxation does respond to densification in USG like the $\alpha$-relaxation. Here, we provide an explanation based on two general properties established from the studies of glasses and liquids at elevated pressures and applied to USG. The increase in density of the glasses formed under high pressure can be even larger than that in USG. One property is the approximate invariance of the ratio $\tau_{\alpha}\left(T_{\text {on }}\right) / \tau_{\beta}\left(T_{\text {on }}\right)$ to density change at constant $\tau_{\alpha}\left(T_{\text {on }}\right)$, and the other is the same $\rho^{\gamma} / T$-dependence of $\tau_{\beta}$ in USG and OG where $\rho$ is the density and $\gamma$ is a material constant. These two properties are derived using the Coupling Model, giving a theoretical explanation of the phenomena. The explanation is also relevant for a full understanding of the experimental result that approximately the same surface diffusion coefficient is found in USG and OG with and without physical aging, and ultrathin films of a molecular glass-former.
\end{abstract}

\section{Introduction}

Glasses produced by vapor deposition on a substrate at an optimal temperature below the bulk glass transition temperature $\left(\sim 0.85 T_{\mathrm{g}}\right.$, where $T_{\mathrm{g}}$ is the glass transition temperature of the material) have density considerably higher than ordinary glasses even after aging for a realistically long time. ${ }^{1-7}$ These glasses are more stable than the ordinary glasses (OG), and hence are called ultrastable glasses (USG). Compared with OG, some dynamic and thermodynamic properties of USG are novel and challenging to explain. ${ }^{8-11}$ One such property and its explanation are the focus of the present paper.

\footnotetext{
${ }^{a}$ CNR-IPCF, Largo Bruno Pontecorvo 3, I-56127, Pisa, Italy.

E-mail: kiangai@yahoo.com,kia.ngai@pi.ipcf.cnr.it

${ }^{b}$ Silesian Center for Education and Interdisciplinary Research, 75 Pulku Piechoty 1, 41-500 Chorzow, Poland. E-mail: crodrigueztinoco@gmail.com

${ }^{c}$ Institute of Physics, University of Silesia, 75 Pulku Piechoty 1, 41-500 Chorzow, Poland

$\dagger$ Electronic supplementary information (ESI) available: Fig. S1-S4. See DOI: $10.1039 / \mathrm{c} 8 \mathrm{cp} 05107 \mathrm{k}$
}

The stability of USG is measured by the onset temperature for the transformation into the supercooled liquid, $T_{\text {on }}$. Its value is significantly higher than $T_{\text {on }}$ or $T_{\mathrm{g}}$ of the ordinary glass (OG) formed by cooling. These changes in $T_{\text {on }}$ imply an increase of the structural $\alpha$-relaxation time, $\tau_{\alpha}$, by many orders of magnitude in USG compared to that in OG at the same temperature. Often observed in OG and USG is not only the $\alpha$-relaxation but also a secondary relaxation. Some, but not all, secondary relaxations have properties strongly connected or related to those of the $\alpha$-relaxation, and hence they are fundamentally important. ${ }^{12}$ These secondary relaxations are called the Johari-Goldstein (JG) $\beta$-relaxations in order to distinguish them from the other trivial ones. ${ }^{12}$ In contrast to the $\alpha$-relaxation time $\tau_{\alpha}$, the JG $\beta$-relaxation time, $\tau_{\beta}$, in USG of toluene, ${ }^{13}$ etoricoxib $^{14}$ and telmisartan ${ }^{15}$ is about an order of magnitude longer than that in OG and has nearly the same activation energy $E_{\beta}$. The data from these three cases are extracted from C. Rodríguez-Tinoco et al. ${ }^{15}$ and shown in Fig. 1 . On the other hand, the secondary relaxations of maltose octa-acetate, carvedilol and celecoxib, also discussed in ref. 15, involve 


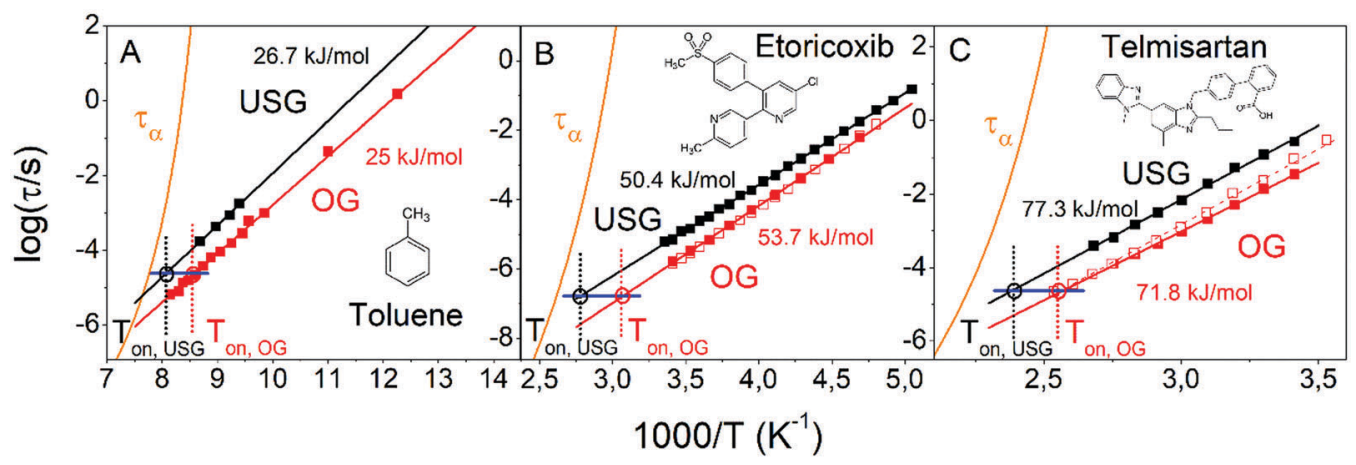

Fig. 1 Adapted from ref. 15. Arrhenius plot of $\tau_{\beta}$ in USG and OG of (A) toluene, (B) etoricoxib, and (C) telmisartan. Black and red closed symbols correspond to the USG and the OG obtained after transformation of the USG and subsequent cooling down, respectively. The red open points and the orange VFT fit to $\tau_{\alpha}$ in $\left(B\right.$ and $C$ ) are from the literature (etoricoxib, ${ }^{14,16,17}$ telmisartan $^{18,19}$ ). All the data of toluene are extracted from Yu et al. ${ }^{13}$ The black and red circles indicate the value of $\tau_{\beta}$ at $T_{\text {on }}$. The horizontal blue lines in graphs a-c bring out the invariance of $\tau_{\beta}$ at $T_{\text {on }}$. The sketch of each molecule is also depicted.

intramolecular degrees of freedom, bear no connection to the $\alpha$-relaxation, ${ }^{15}$ do not belong to the class of JG $\beta$-relaxations, and are not considered in this paper.

On the other hand, the relaxation strength of the JG $\beta$-relaxation, $\Delta \varepsilon_{\beta}$, is significantly reduced in USG in toluene to such a level that it would require several thousands of years of annealing for an ordinary glass to achieve the same state. ${ }^{13-15}$ The reduction of $\Delta \varepsilon_{\beta}$ indicates that the JG $\beta$-relaxation does respond to densification in the USG like the $\alpha$-relaxation. Notwithstanding, the much smaller changes of $\tau_{\beta}$ and $E_{\beta}$ by densification in USG are in stark contrast to the behavior of the $\alpha$-relaxation time $\tau_{\alpha}$, and this remarkable experimental observation deserves an explanation. In this paper, we provide an explanation based on two general properties established from the studies of glasses and liquids at elevated pressures ${ }^{20-27}$ and applied it to USG. The increase in density of the glasses formed under high pressure can be even larger than that in USG, and hence the general properties should apply. One of the properties deduced and applied to USG is the approximate invariance of the ratio $\tau_{\alpha}\left(T_{\mathrm{on}}\right) / \tau_{\beta}\left(T_{\mathrm{on}}\right)$ to density change at constant $\tau_{\alpha}\left(T_{\text {on }}\right)$, and the other is the same $\rho^{\gamma} / T$-dependence of $\tau_{\beta}$ in USG and OG where $\rho$ is the density and $\gamma$ is a material constant. Furthermore, we show how these two general properties can be derived theoretically by applying the Coupling Model, ${ }^{28}$ and by this application we have completed our explanation of the observed minor changes of $\tau_{\beta}$ and $E_{\beta}$ by densification in USG.

The explanation is also relevant in achieving a better understanding than before ${ }^{29}$ of another remarkable experimental finding, which is about the same surface diffusion coefficient, $D_{\mathrm{S}}$, in USG and OG with and without physical aging, and ultrathin films of the molecular glass, $N, N^{\prime}$-bis(3-methylphenyl)- $N, N^{\prime}$-diphenylbenzidine (TPD) by Fakhraai and coworkers. ${ }^{30-32}$

\section{Two general properties applied to USG}

As mentioned in the Introduction, to explain the properties of the JG $\beta$-relaxation in USG compared with the OG we shall apply two general dynamic and thermodynamic properties of the $\alpha$-relaxation and JG $\beta$-relaxation established before from studies at elevated pressures in molecular glass-formers. ${ }^{20-27}$ The application is justified by the fact that density is increased at elevated pressure, and the increase can be comparable to or even larger than that in USG. Through the application, we are able to explain why the Johari-Goldstein (JG) $\beta$-relaxation time, $\tau_{\beta}$, in USG of toluene, ${ }^{13}$ etoricoxib ${ }^{14}$ and telmisartan ${ }^{15}$ is about an order of magnitude longer than that in OG, and has nearly the same activation energy $E_{\beta}$. Later we shall show how the two general properties are derived from theoretical considerations. Thus, a theoretical explanation of the properties of the JG $\beta$-relaxation in USG is obtained.

\section{A. General property leading to the same $\tau_{\beta}\left(T_{o n}\right)$ for USG and OG}

By elevating pressure $P$ to hundreds of MPa or more, one can increase the glass transition temperature from $T_{\mathrm{g}}$ at ambient pressure, $P_{0}=0.1 \mathrm{MPa}$, of many molecular glass-formers by several tens of degrees to $T_{\mathrm{g} P}$ and the density by a few per cent. These changes are comparable to or larger than those achieved by producing USG. Found in general in many molecular glassformers ${ }^{20-28}$ is the approximate invariance of $\tau_{\beta}(T, P)$ to variations of $P$ and $T$ provided $\tau_{\alpha}(T, P)$ are kept constant. Examples of experimental evidence for this property in OG are shown in Fig. S1 and S2 in the ESI. $\dagger$ Since $\tau_{\alpha}\left(T_{\mathrm{g} P}, P\right)$ are approximately the same at $T_{\mathrm{g} P}$ for all $P$ by definition of glass transition temperature, the property specified at $T_{\mathrm{g} P}$ states that $\tau_{\beta}\left(T_{\mathrm{g} P}, P\right)$ is invariant to variations of $P$. In turn, this can be restated as approximate invariance of $\tau_{\beta}\left(T_{\mathrm{g} P}, \rho_{\mathrm{g} P}\right)$ to variations of $\rho_{\mathrm{g} P}$ in OG, where $\rho_{\mathrm{g} P}$ is the density at temperature equal to $T_{\mathrm{g} P}$ and pressure $P$ determined from the equation of state connecting $P, T$, and specific volume $V$ or density $\rho$ of the glass-former. Pressure $P$ is just a parameter used to vary $T_{\mathrm{g} P}$ and $\rho_{\mathrm{g} P}$ to bring out the property of the invariance of $\tau_{\beta}\left(T_{\mathrm{g} P}, \rho_{\mathrm{g} P}\right)$. It can be replaced by density $\rho$ in some cases where $\rho$ is changed directly by some operation instead of applied pressure. Producing USG by vapor deposition is such an operation whereby the density is increased compared to OG. The equivalence of effects due to change in density either by the route of USG or by application of pressure on OG can be appreciated from the fact that $T_{\text {on }}$ of the 
USG of indomethacin is larger than that of the OG at ambient pressure, but $T_{\text {on }}$ of the two glasses increases with pressure and becomes nearly the same upon reaching $300 \mathrm{MPa}{ }^{33}$ Thus, the approximate invariance of $\tau_{\beta}\left(T_{\mathrm{g} P}, \rho_{\mathrm{g} P}\right)$ to variations of $\rho_{\mathrm{g} P}$ translates to the approximate invariance of $\tau_{\beta}\left(T_{\text {on }}\right)$ with the change from OG to USG. It is expressed explicitly by

$$
\tau_{\beta, \mathrm{USG}}\left(T_{\mathrm{on}, \mathrm{USG}}\right) \approx \tau_{\beta, \mathrm{OG}}\left(T_{\mathrm{on}, \mathrm{OG}}\right),
$$

after replacing $T_{\mathrm{gP}}$ by $T_{\text {on,USG }}$ for USG and $T_{\text {on,OG }}$ for OG. Thus, this property found in USG and OG of toluene, ${ }^{13}$ etoricoxib ${ }^{14}$ and telmisartan ${ }^{15}$ is a special application of the general property of the approximate invariance of $\tau_{\beta}\left(T_{\mathrm{g} P}, P\right)$ to variations of $P$ or equivalently the invariance of $\tau_{\beta}\left(T_{\mathrm{g} P}, \rho_{\mathrm{g} P}\right)$ to variations of $\rho_{\mathrm{g} P}$ in OG. We shall provide the theoretical justification of the general property in Section (C) after the presentation of the other general property in Section (B) from which we can rationalize why the Arrhenius temperature dependence of $\tau_{\beta, \text { USG }}$ in USG is not much different from that of $\tau_{\beta, \mathrm{OG}}$ in OG.

\section{B. General property leading to similar Arrhenius $T$-dependence of $\tau_{\beta, \text { USG }}$ and $\tau_{\beta, O G}$}

The structural $\alpha$-relaxation time, $\tau_{\alpha}$, of non-associated small molecular and polymeric glass-formers obeys thermodynamic scaling, i.e. $\tau_{\alpha}$ is a function of the product variable, $\rho^{\gamma} / T$, where $\gamma$ is a material dependent parameter. ${ }^{34-36}$ This property in many glass-formers was deduced from measurements of $\tau_{\alpha}$ at ambient and elevated pressures analyzed in conjunction with $P-V-T$ data. The property of the invariance of the ratio $\tau_{\alpha}(T, P) / \tau_{\beta}(T, P)$ to variations of $T$ and $P$ while keeping $\tau_{\alpha}(T, P)$ constant $^{20-27}$ is key to arriving at the results in Section (A). It also has the immediate consequence that $\tau_{\beta}$ is also a function of $\rho^{\gamma} / T$, if $\tau_{\alpha}$ is a function of $\rho^{\gamma} / T$, although the two functions $\tau_{\beta}=f_{\beta}\left(\rho^{\gamma} / T\right)$ and $\tau_{\alpha}=F_{\alpha}\left(\rho^{\gamma} / T\right)$ are different. This is an example of the dynamic and thermodynamics properties of the JG $\beta$-relaxation similar to those of the $\alpha$-relaxation, ${ }^{20-27}$ which follows from the approximate relation, ${ }^{23,27} \tau_{\alpha}\left(\rho^{\gamma} / T\right) \approx\left[t_{\mathrm{c}}^{\beta_{\mathrm{KWw}}-1} \tau_{\beta}\left(\rho^{\gamma} / T\right)\right]^{1 / \beta_{\mathrm{KWw}}}$, where $t_{\mathrm{c}}(\sim 1 \mathrm{ps})$ is a constant and $\beta_{\mathrm{KWw}}$ is the stretch exponent of the Kohlrausch-Williams-Watts correlation function for the $\alpha$ process. From this approximate relation and the fact that the JG $\beta$-relaxation occurs before the $\alpha$-relaxation in time (i.e., its precursor), it follows that the $\rho^{\gamma} / T$-dependence of $\tau_{\alpha}=F_{\alpha}\left(\rho^{\gamma} / T\right)$ originates from $\tau_{\beta}=f_{\beta}\left(\rho^{\gamma} / T\right)^{27}$ as required by causality. Another piece of evidence for $\rho^{\gamma} / T$-dependence originating from $\tau_{\beta}=f_{\beta}\left(\rho^{\gamma} / T\right)$ can be drawn from the remarkably small value of $r_{\mathrm{s}}$ at which the steepness of the repulsive part of the intermolecular potential $U(r)$ determines the scaling exponent $\gamma{ }^{27}$ The short distance $r_{\mathrm{s}}$ was found by molecular dynamics simulations of several Lennard-Jones liquids ${ }^{37}$ and in cis 1,4-polybutadiene as shown in Fig. S3 in the ESI, $\uparrow^{38}$ and reviewed in ref. 27.

The result, $\tau_{\beta}=f_{\beta}\left(\rho^{\gamma} / T\right)$, is the general property we now use to deduce why the Arrhenius temperature dependence of $\tau_{\beta}$ in USG is not much different from that in OG. Although the $\rho^{\gamma} / T$-dependences of $\tau_{\alpha}=F_{\alpha}\left(\rho^{\gamma} / T\right)$ and $\tau_{\beta}=f_{\beta}\left(\rho^{\gamma} / T\right)$ are mostly considered in the liquid state, it is applicable also in the glassy state. An example demonstrating this is the study of diglycidyl ether of bisphenol-A (DGEBA) with $M_{\mathrm{w}}=380 \mathrm{~g} \mathrm{~mol}^{-1} \cdot{ }^{23}$ In the glassy state, the temperature dependence of $f_{\beta}\left(\rho^{\gamma} / T\right)$ becomes effectively Arrhenius. The difference between the activation energy $E_{\beta, \text { USG }}$ in USG and $E_{\beta, \mathrm{OG}}$ in OG comes from the factor $\rho^{\gamma}$ and the difference between the density $\rho_{\text {USG }}$ of USG and $\rho_{\mathrm{OG}}$ of OG. Hence the ratio $E_{\beta, \mathrm{USG}} / E_{\beta, \mathrm{OG}}$ can be estimated from the value of $\left(\rho_{\mathrm{USG}} / \rho_{\mathrm{OG}}\right)^{\gamma}$. Ultrastable glasses of IMC have a density up to $1.4 \%$ more than that of the liquid-cooled $\mathrm{OG},{ }^{4}$ and a similar value is obtained for USG of TPD. ${ }^{39}$ The ratio $E_{\beta, \mathrm{USG}} / E_{\beta, \mathrm{OG}}$ is equal to $(1.014)^{\gamma}$. Typical values of $\gamma$ fall within the range of 2 to 7 , and toluene has $\gamma=7$. The largest value of $E_{\beta, \mathrm{USG}} / E_{\beta, \mathrm{OG}}=(1.014)^{\gamma}$ is 1.1 for $\gamma=7$. In other words, $E_{\beta, \mathrm{USG}}$ is larger than $E_{\beta, \mathrm{OG}}$ by merely $10 \%$, and therefore the small increase of activation energy in USG compared to OG is a consequence of the general property of the $\rho^{\gamma} / T$-dependence of $\tau_{\beta}$.

There are other experimental results that prove the fact that the $\rho^{\gamma} / T$-dependence of $\tau_{\alpha}$ and $\tau_{\beta}$ is the same in both USG and OG because the repulsive part of the intermolecular potential is unchanged. Wide-angle X-ray scattering (WAXS) experiments were performed on USG and OG of indomethacin $(\mathrm{IMC})^{3}$ and etoricoxib. ${ }^{14}$ In both cases, the intensity as a function of the scattering wave vector $q$ exhibits no difference at $q$ values higher than that of the peak, indicating that the relation of $g(r)$ with the repulsive part of the intermolecular potential is the same for USG and OG. Moreover, molecular dynamics simulations of $\mathrm{USG}^{40,41}$ show that the changes of its $g(r)$ from that of OG occur at larger values of $r$, which are irrelevant for the determination of $\gamma$ from the slope of the repulsive part of the intermolecular potential. Therefore, from these two facts we conclude the same repulsive part of the potential, and hence the same $\rho^{\gamma} / T$-dependence of $\tau_{\beta}$ for USG and OG. In a recent report, ${ }^{7}$ the authors used the calorimetric transformation time of glasses with different thermodynamic stability to infer the structural relaxation times $\tau_{\alpha}$ of the glassy systems. Interestingly, they found that for all the glasses, USG and OG, the relaxation data have the same $\rho^{\gamma} / T$-dependence, with a unique $\gamma$ exponent (see Fig. S4 in ESI $\dagger$ ). This is another indication that $\gamma$ is practically the same in USG and OG.

\section{Derivation of the two general properties to complete the explanation}

The two general properties of the JG $\beta$-relaxation time $\tau_{\beta}$ and its relation to $\tau_{\alpha}$ have been applied to compare USG with OG in Sections (A) and (B) with two results: (i) the value of $\tau_{\beta, \mathrm{USG}}(T)$ at $T=T_{\text {on,USG }}$ is the same as $\tau_{\beta, \mathrm{OG}}(T)$ at $T=T_{\text {on,OG }}$, i.e., eqn (1), and (ii) the activation energy $E_{\beta, \mathrm{USG}}$ of the Arrhenius temperature dependence of $\tau_{\beta, \text { USG }}(T)$ for $T<T_{\text {on,USG }}$ is only slightly increased from $E_{\beta, \mathrm{OG}}$ of $\tau_{\beta, \mathrm{OG}}(T)$ for $T<T_{\text {on,OG. }}$. The two results combined explain why the difference between $\tau_{\beta, \mathrm{USG}}(T)$ and $\tau_{\beta, \mathrm{OG}}(T)$ over the entire common temperature range below $T_{\text {on,OG }}$ is minor as observed experimentally in toluene, ${ }^{13}$ etoricoxib, ${ }^{14}$ and telmisartan. ${ }^{15}$ However, the explanation is not complete until we have given the theoretical basis of the two general properties. This is our next task and we use the Coupling Model (CM). 
The CM starts from the primitive relaxation which is independent and local. The time honored CM equation, ${ }^{27,28}$

$$
\tau_{\alpha}(T, P)=\left[t_{\mathrm{c}}^{-n(T, P)} \tau_{0}(T, P)\right]^{1 /[1-n(T, P)]}
$$

links the primitive relaxation time $\tau_{0}(T, P)$ to the cooperative many-molecule $\alpha$-relaxation. In eqn (2), $t_{\mathrm{c}}$ is the onset time of classical $\operatorname{chaos}^{28}$ and its magnitude is about 1 to 2 ps for molecular glass-formers and polymers, and was directly determined by quasielastic neutron scattering experiments and molecular dynamics simulations. ${ }^{28}$ The parameter $n(T, P)$ is the fractional exponent of the Kohlrausch correlation function,

$$
\varphi_{\mathrm{K}}(t)=\exp \left[-\left[\frac{t}{\tau_{\alpha}(T, P)}\right]^{1-n(T, P)}\right] .
$$

The independent and local nature of the primitive relaxation suggests that it is similar to the JG $\beta$-relaxation. The two are not identical because the JG $\beta$-relaxation is composed of a distribution of processes ${ }^{42,43}$ and the primitive relaxation is only the leading part. Notwithstanding, approximate correspondence of the two relaxation times $\tau_{0}(T, P)$ and $\tau_{\beta}(T, P)$ is expected and predicted. ${ }^{27,28,44}$ Written as

$$
\tau_{\beta}(T, P) \approx \tau_{0}(T, P),
$$

this relation has been verified in many glass-formers of different types, ${ }^{20-28,44}$ where the JG $\beta$-relaxation is resolved and its loss peak frequency provides a direct and unequivocal determination of $\tau_{\beta}(T, P)$. In many of these cases, the value of $\tau_{\beta}(T, P)$ differs from $\tau_{0}(T, P)$ calculated by eqn (4) within one order of magnitude or less.

The CM is based on classical chaos engendered by the anharmonic intermolecular potential. ${ }^{28,45}$ In the model, it is the intermolecular potential in conjunction with the primitive relaxation time $\tau_{0}(T, P)$ which exclusively determines $\varphi_{\mathrm{K}}(t)$. Upon varying $P$ and $T$ while keeping $\tau_{0}(T, P)$ the same, the intermolecular potential is also unchanged, ${ }^{37,38,46}$ therefore $\varphi_{\mathrm{K}}(t)$ is the same, or both $\tau_{\alpha}(T, P)$ and $n(T, P)$ are the same. Therefore, the exact co-invariance to changes of $P$ and $T$ of the three quantities, $\tau_{0}(T, P), \tau_{\alpha}(T, P)$ and $n(T, P)$, is immediately the consequence of the CM. Combining this property with $\tau_{\beta}(T, P) \approx \tau_{0}(T, P)$, we have derived the approximate co-invariance of $\tau_{\beta}(T, P), \tau_{\alpha}(T, P)$ and $n(T, P)$. From this result, the general property used in Section (A) (i.e., approximate invariance of $\tau_{\beta}(T, P)$ to variations of $P$ and $T$ at constant $\left.\tau_{\alpha}(T, P)\right)$ is now derived from the $\mathrm{CM}$. The reader may recall the application of this general property to USG and OG in showing that $\tau_{\beta, \mathrm{USG}}\left(T_{\text {on,USG }}\right)$ is approximately equal to $\tau_{\beta, \mathrm{OG}}\left(T_{\text {on,OG }}\right){ }^{14,15}$

Derived also from the CM is the invariance of $n(T, P)$ or the $\alpha$-relaxation frequency dispersion to variations of $P$ and $T$ at constant $\tau_{\alpha}(T, P)$, which has not been utilized in this paper in the relation between USG and OG. Nevertheless, it is worthwhile to point out that it is a remarkable prediction. Equally remarkable is that this property has been verified in so many glass-formers, molecular or polymeric. ${ }^{47,48}$ The importance of the frequency dispersion is brought out by the property.
In Section (B) we use another property and give a reason to show that the Arrhenius activation energies $E_{\beta, \text { USG }}$ of USG and $E_{\beta, \text { OG }}$ of OG are not much different. The property is that $\tau_{\beta}$ is a function $f\left(\rho^{\gamma} / T\right)$ of $\rho^{\gamma} / T$ with the same $\gamma$ as the function $F\left(\rho^{\gamma} / T\right)$ of $\tau_{\alpha}$. The reason is that $\gamma$ is determined by the repulsive part of the intermolecular potential which is the same in USG and OG, and hence the same $f\left(\rho^{\gamma} / T\right)$ for USG and OG. We have demonstrated in Section (B) that the property, $\tau_{\beta}$ a function of $\rho^{\gamma} / T$, is a consequence of the approximate invariance of the ratio $\tau_{\alpha}(T, P) / \tau_{\beta}(T, P)$ to variations of $T$ and $P$ while keeping $\tau_{\alpha}(T, P)$ constant. The latter is just part of the approximate coinvariance of $\tau_{\beta}(T, P), \tau_{\alpha}(T, P)$ and $n(T, P)$ derived above from the $\mathrm{CM}$, and hence the property is justified as well.

\section{Accounting for the minor difference in $\tau_{\beta}(T)$ between USG and $O G$}

Having derived in (C) the two properties in (A and B), we are ready to put them together to account for the minor change of $\tau_{\beta}(T)$ in USG compared to OG observed experimentally. The change is given by the ratio, $\tau_{\beta, \mathrm{USG}}(T) / \tau_{\beta, \mathrm{OG}}(T)$, which we only need to calculate at $T=T_{\text {on,OG }}$ because the activation energies $E_{\beta, \text { USG }}$ and $E_{\beta, \mathrm{OG}}$ of the Arrhenius $T$-dependences of $\tau_{\beta, \mathrm{USG}}(T)$ and $\tau_{\beta, \mathrm{OG}}(T)$ in the glassy states are about the same. Since the ratio is the main interest, it is denoted by $\hat{R}$ and written explicitly as

$$
\hat{R} \equiv \tau_{\beta, \mathrm{USG}}\left(T_{\mathrm{on}, \mathrm{OG}}\right) / \tau_{\beta, \mathrm{OG}}\left(T_{\text {on, OG }}\right)
$$

Upon combining eqn (5) with $\tau_{\beta, \mathrm{USG}}\left(T_{\mathrm{on}, \mathrm{USG}}\right) \approx \tau_{\beta, \mathrm{OG}}\left(T_{\mathrm{on}, \mathrm{OG}}\right)$, i.e., eqn (1), we have the result $\hat{R} \approx\left[\tau_{\beta, \mathrm{USG}}\left(T_{\text {on,OG }}\right) / \tau_{\beta, \mathrm{USG}}\left(T_{\text {on,USG }}\right)\right]$, which can be calculated from the change of the factor, $\exp \left(E_{\beta, \mathrm{USG}} / R T\right)$, of the temperature dependence of $\tau_{\beta, \mathrm{USG}}(T)$. The final result is

$$
\hat{R}=\exp \left[\Lambda\left(1-\frac{T_{\text {on }, \mathrm{OG}}}{T_{\text {on, }, \mathrm{USG}}}\right)\right],
$$

where $E_{\beta, \mathrm{USG}} / R T_{\text {on,OG }} \equiv \Lambda$. In the case of toluene, by taking $T_{\text {on, OG }}=117 \mathrm{~K}, T_{\text {on,USG }}=124 \mathrm{~K},{ }^{13}$ and $\Lambda=28.5$, we have $\log _{10}(\hat{R})=0.7$. It is slightly less than a decade, and in approximate agreement with the experimental data of Yu et al. ${ }^{13}$

For etoricoxib, with $E_{\beta}=51 \mathrm{~kJ} \mathrm{~mol}^{-1}, T_{\text {on, OG }}=327 \mathrm{~K}, T_{\text {on, USG }}=$ $360 \mathrm{~K}$, and $\Lambda=18.76,{ }^{14}$ we have $\log _{10}(\hat{R})=0.75$, which compares well with the experimental value of $\sim 0.84$ at $T=293 \mathrm{~K}$. The small value of $\Lambda$ for etoricoxib is because the secondary relaxation is not the usual JG $\beta$-relaxation. ${ }^{14,17}$ Notwithstanding, this secondary relaxation has some of the properties of the JG $\beta$-relaxation such as a significant dependence of its relaxation time on pressure. This is the reason why it is used for comparison between USG and OG since the usual JG $\beta$-relaxation of etoricoxib is not resolved.

The difference between $T_{\text {on,USG }}$ of USG and $T_{\text {on,OG }}$ of OG is 18 degrees for TPD. ${ }^{39}$ Taking $T_{\text {on,OG }}=330 \mathrm{~K}$ and $T_{\text {on,USG }}=348 \mathrm{~K}$, and assuming $E_{\beta} / R T_{\text {on,OG }} \equiv \Lambda=24$, the predicted $\log _{10}(\hat{R})=0.54$. This small increase of $\tau_{\beta, \text { USG }}(T)$ in USG of TPD with reference to $\tau_{\beta, \mathrm{OG}}(T)$ of $\mathrm{OG}$ is consistent with the CM explanation ${ }^{29}$ of the invariant surface diffusion observed in the two glasses by Fakhraai and coworkers. ${ }^{31,32}$ 


\section{Discussion}

The main objective of this paper is to explain why the differences in magnitude and temperature dependence of $\tau_{\beta}$ between USG and OG are minor compared with the huge change of $\tau_{\alpha}$. Our goal is accomplished by the theoretical treatment given in Section 1. The explanation is beneficial to complete a previous rationalization of another remarkable experimental finding, ${ }^{29}$ which is approximately the same surface diffusion coefficient $D_{\mathrm{S}}$ in USG, OG, and nanometer thin films of the same glassformer, ${ }^{30-32}$ while their structural $\alpha$-relaxation times differ by many orders of magnitude. The rationalization is based on the relation $D_{\mathrm{S}}(T) \approx d^{2} / 4 \tau(T)$, where $d$ is the size of the molecule, used previously to account for the enhancement of the surface diffusion $^{29}$ (further support for the use of this expression is shown in the ESI, $\dagger$ Fig. S5), and the experimental evidence showing that $\tau_{\beta}(T)$ is approximately the same in USG, aged OG, OG, and ultrathin films. The development in the present paper justifies on theoretical grounds the rationalization given before.

Glass with density higher than USG can be realized by applying pressure $P$ much higher than ambient pressure $P_{0}=0.1 \mathrm{MPa}$. If the minor changes in the magnitude and activation energy of $\tau_{\beta}$ in USG compared to OG is general, it should be observed by elevating pressure from $P_{0}$ to $P$. The answer is positive from the results of studies at elevated pressures. ${ }^{20-28} \mathrm{An}$ example is the isobaric data of DGEBA (diglycidyl ether of bisphenol-A, $M_{\mathrm{w}}=380 \mathrm{~g} \mathrm{~mol}^{-1}$, also known as EPON 828). Shown in Fig. 2 are the $\alpha$-relaxation times $\tau_{\alpha}$ and JG $\beta$-relaxation times $\tau_{\beta}$ at ambient pressure $P_{0}$ of $0.1 \mathrm{MPa}$ and at $P=400 \mathrm{MPa}$.

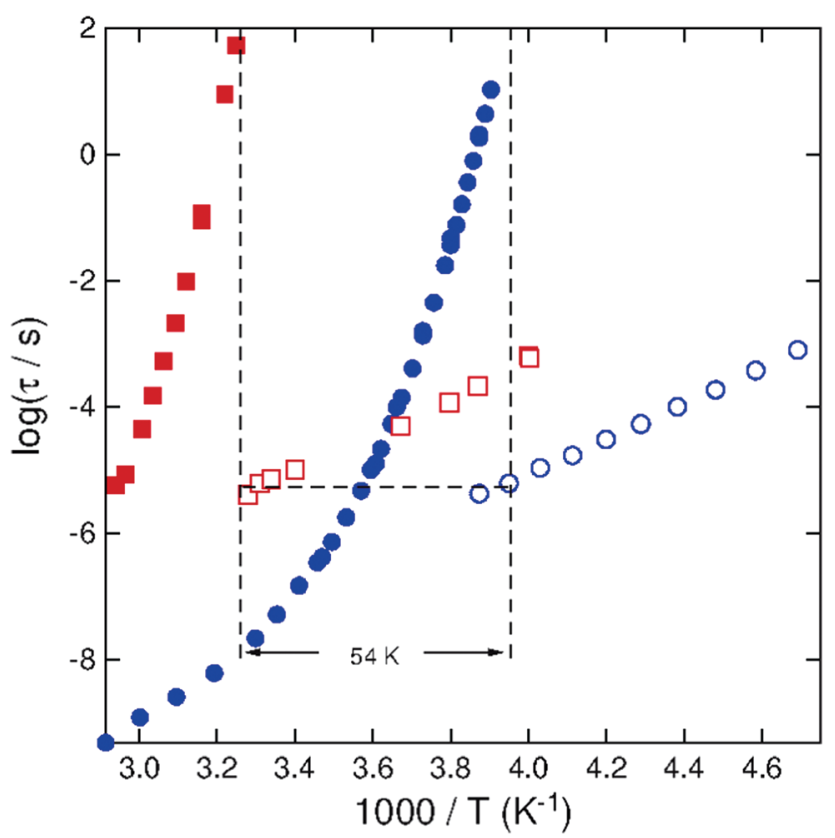

Fig. 2 Logarithm of characteristic time of dielectric loss maximum of DGEBA (diglycidyl ether of bisphenol-A, $M_{\mathrm{w}}=380 \mathrm{~g} \mathrm{~mol}^{-1}$, also known as EPON 828 ) for the $\alpha$-relaxation and JG $\beta$-relaxation under isobaric conditions versus reciprocal temperature. Red symbols, $P=400 \mathrm{MPa}$; blue symbols, $P_{0}=0.1 \mathrm{MPa}$. The difference in $T_{\mathrm{g}}$ is $54 \mathrm{~K}$, which is matched by the separation of the JG $\beta$-relaxation time as indicated by the dashed line.
Defining glass transition temperatures by $\tau_{\alpha}=100 \mathrm{~s}$, we have $T_{\mathrm{g}}=252.8 \mathrm{~K}$ at $P_{0}$ and $T_{\mathrm{g} P}=306.7 \mathrm{~K}$ at $P=400 \mathrm{MPa}$. The increase of $54 \mathrm{~K}$ is significantly larger than that expected for USG. The value of $\tau_{\beta}\left(T_{\mathrm{g}}, P_{0}\right)$ is about the same as $\tau_{\beta}\left(T_{\mathrm{g} P}, P\right)$. The activation energy is $E_{\beta}=52.2 \mathrm{~kJ} \mathrm{~mol}^{-1}$ at ambient pressure, and it is about the same as the value at $P=400 \mathrm{MPa}$. Thus, the relation of $\tau_{\beta}$ at high pressure to ambient pressure is the same as that of USG to OG. The ratio $\tau_{\beta}(T, P) / \tau_{\beta}\left(T, P_{0}\right) \approx 100$ is larger, and this is consistent with the density increase by elevating pressure, although the value of $\gamma$ of DGEBA is 3.5, which is a factor of 2 smaller than that of toluene.

In this paper we have exclusively considered toluene, etoricoxib, and telmisartan with regard to the change of their secondary relaxations upon densification in USG compared to OG. The properties of the secondary relaxation in these three glass-formers are connected to or correlated with that of the $\alpha$-relaxation, as discussed before in ref. 15 . They belong to the class of JG $\beta$-relaxations if the relaxation time $\tau_{\beta}(P, T)$ satisfies the criteria set forth in ref. 12 and 44, including the approximate relation $(4)$, i.e. $\tau_{\beta}(P, T) \approx \tau_{0}(P, T)$. This criterion together with the CM eqn (2) led to the approximate relation,

$$
\tau_{\alpha}(P, T) \approx\left[t_{\mathrm{c}}^{-n} \tau_{\beta}(P, T)\right]^{1 /(1-n)} .
$$

From this relation, another criterion for JG $\beta$-relaxation is that the $P, T$ and $\rho$ dependences of $\tau_{\beta}(P, T, \rho)$ are correlated with those of $\tau_{\alpha}(P, T, \rho)$ approximately. The $\tau_{\beta}(P, T, \rho)$ of toluene, etoricoxib, and telmisartan increase upon densification in USG compared to OG in concert with $\tau_{\alpha}(P, T, \rho)$, indicating that the secondary relaxations are the JG $\beta$-relaxations.

The secondary relaxation with properties uncorrelated with those of the $\alpha$-relaxation and that does not satisfy the criteria is unimportant and is referred to as a non-JG relaxation. This is the case for the well-resolved secondary $\delta$-relaxation of celecoxib, and $\gamma$-relaxations of D-maltose octa-acetate and carvedilol, discussed also in ref. 15. Their $\tau_{\gamma}(T, \rho)$ becomes faster while $\tau_{\alpha}(T, \rho)$ becomes slower on densification of the glass by vapour deposition. Thus, the change of $\tau_{\gamma}(T, \rho)$ upon densification is opposite to that of $\tau_{\alpha}(T)$, and hence the $\gamma$-relaxations in D-maltose octaacetate and carvedilol are the non-JG relaxations. For celecoxib, it was shown before ${ }^{49}$ that it is the fast $\delta$-relaxation, and its relaxation time $\tau_{\delta}$ is much shorter than the calculated primitive relaxation time $\tau_{0} \approx \tau_{\beta}$. The JG $\beta$-relaxation is present but it has low dielectric strength and is detected as a broad shoulder in the glassy state. Its relaxation time $\tau_{\beta}$ is in agreement with $\tau_{0}$. It was not considered in ref. 15 . For D-maltose octa-acetate and carvedilol, the non-JG nature of the resolved secondary relaxation is further justified by the fact that the observed relaxation time $\tau_{\gamma}(T)$ is much shorter than $\tau_{0}(T)$ calculated by eqn (2). The JG $\beta$-relaxation of D-maltose octa-acetate and carvedilol cannot be resolved due to its proximity to the $\alpha$-relaxation. Nevertheless, this is irrelevant to this work because we are considering the resolved JG $\beta$-relaxations of toluene, etoricoxib, and telmisartan in the change by densification in USG.

Some of the results in Sections (B) and (C) are based on the approximate invariance of $\tau_{\beta}(T, P)$ to variations of $T$ and $P$ while keeping $\tau_{\alpha}(T, P)$ constant, which follows from relation (7). 
Only approximate invariance of $\tau_{\beta}(P, T)$ is predicted because the JG $\beta$-relaxation is composed of a distribution of processes involving increasing number of molecules with increasing time, ${ }^{42,43}$ and the value of $\tau_{\beta}(P, T)$ determined from experimental data for different combinations of $P$ and $T$ may not be associated with the same process in the distribution. Fortunately, in all previous experiments involving non-polymeric glass-formers in ref. 15 and $20-28$, the JG $\beta$-relaxation was resolved in the dielectric spectra as a pronounced loss peak, and the peak frequency $f_{\beta}(P, T)$ provides a characteristic time $\tau_{\beta}(P, T)$ of the distribution. No fit is needed to determine $f_{\beta}(P, T)$ and test the approximate invariance. Nearly exact invariance of $f_{\beta}(P, T)$ or $\tau_{\beta}(P, T)$ was found in all cases published in ref. 15 and 20-28 as well as in DGEBA (diglycidyl ether of bisphenol-A, $M_{\mathrm{w}}=380 \mathrm{~g} \mathrm{~mol}^{-1}$ ) shown in Fig. 2, and thus overwhelmingly verifying the approximate invariance of $\tau_{\beta}(P, T)$ for molecular glass-formers.

On the other hand, the dielectric spectra of the polymers, polyisoprene, $^{43}$ polymethylmethacrylate $^{50}$ (PMMA), and 1,4 polybutadiene, ${ }^{51}$ are not as ideal or straightforward as in the case of molecular glass-formers to test invariance of $\tau_{\beta}(P, T)$. The $\alpha$-relaxation of PMMA has low dielectric strength and its loss peak is not well resolved for many $P$ and $T$ combinations. Consequently, an assumption of the frequency dispersion of the $\alpha$-relaxation had to be made, ${ }^{50}$ and used to fit the loss spectra with further assumption that the $\alpha$-relaxation and the JG $\beta$-relaxation represented by a Cole-Cole function are additive. It is remarkable but questionable that practically the same value of $\tau_{\alpha}(P, T)$ was obtained from the fits for different $P$ and $T$ despite the lack of well defined $\alpha$-loss peaks. Nevertheless, the deviation of the deduced values of $\tau_{\beta}(P, T)$ from exact invariance is only \pm half a decade, while the $\tau_{\alpha}(P, T)$ values change over 7 orders of magnitude. The results therefore are consistent with the approximate invariance of $\tau_{\beta}(P, T)$ predicted from relation (7).

In the case of 1,4 polybutadiene ${ }^{51}$ the data at ambient pressure of $0.1 \mathrm{MPa}$ show a long plateau or a broad shoulder, and $f_{\beta}(P, T)$ was deduced from the fit by assuming the Cole-Cole function representing the JG $\beta$-relaxation and the data can be represented by the sum of the Cole-Cole function and the $\alpha$-loss represented by the Fourier transform of the Kohlrausch function with its intensity adjusted in the global fit. This is common practice, but no one can be sure whether the JG $\beta$-relaxation is a Cole-Cole function and the assumption of additivity is correct or not, and thus the value of $\tau_{\beta}(P, T)$ deduced for $0.1 \mathrm{MPa}$ is debatable. The same fits were made to data taken at elevated pressures, where the JG $\beta$-relaxation shows up as broad loss peaks. Despite the loss peaks at elevated pressures being very broad, the loss peak frequencies $f_{\beta}(P, T)$ are approximately the same. Thus, except for the questionable value of $\tau_{\beta}(P, T)$ at ambient pressure deduced by fitting data without a $\beta$-loss peak, the peak frequencies of data at elevated pressures are approximately the same and consistent with the predicted approximate invariance of $\tau_{\beta}(P, T)$. Only studies of other polymers having both well resolved $\alpha$ and $\beta$ loss peaks in the future, as in the molecular glass-formers, can critically test the approximate invariance of $\tau_{\beta}(P, T)$ for polymers. The present paper deals exclusively with molecular glass-formers, toluene, etoricoxib, and telmisartan. Since all data of molecular glassformers in ref. 15 and 20-28, as well as those of DGEBA shown in Fig. 2, show consistency with the approximate invariance of $\tau_{\beta}(P, T)$, the unsettled issue of polymers are not relevant for the present paper.

\section{Conclusions}

The onset temperature $T_{\text {on }}$ of ultrastable glass (USG) is significantly higher than that of ordinary glass (OG) due to the increase in density and this suggests an increase in the $\alpha$-relaxation time $\tau_{\alpha}$ by many orders of magnitude. The JG $\beta$-relaxation also responds to the higher density by a large reduction in relaxation strength, which would take thousands of years of aging for OG to achieve. However, experimental studies of USG and OG show the increase of the relaxation time $\tau_{\beta}(T)$ of USG compared to OG is within one decade over the entire common temperature range. Thus, the activation energy of $\tau_{\beta}(T)$ is practically the same in the two glasses. The insensitivity of these dynamic properties of the JG $\beta$-relaxation to the density increase in USG is remarkable and challenging to explain. We have successfully explained these properties by the Coupling Model (CM) after considering that the intermolecular potential in USG remains the same as in OG. High pressure can facilitate the formation of glass with higher density than at ambient pressure, and we demonstrate that the change in properties of $\tau_{\beta}$ is similar to that in USG compared to OG. This proves that the effects are general and the explanation is given. The explanation also applies to another remarkable finding of approximately the same surface diffusion coefficient $D_{\mathrm{S}}$ in USG, OG, and nanometer thin films of the same glass former. ${ }^{29-32}$

\section{Conflicts of interest}

There are no conflicts to declare.

\section{Acknowledgements}

C. R.-T. and M. P. acknowledge the support from the National Science Centre through the Polonez scheme (Grant No. DEC2015/19/P/ST3/03540/2). This project has received funding from the European Union's Horizon 2020 research and innovation programme under the Marie Skłodowska-Curie grant agreement No. 665778.

\section{References}

1 S. F. Swallen, K. L. Kearns, M. K. Mapes, Y. S. Kim, R. J. McMahon, M. D. Ediger, T. Wu, L. Yu and S. Satija, Organic Glasses with Exceptional Thermodynamic and Kinetic Stability, Science, 2007, 315, 353-356.

2 E. Leon-Gutierrez, A. Sepúlveda, G. Garcia, M. T. ClavagueraMora, J. Rodríguez-Viejo, T. Matsuo, L. H. Allen, T. L. Yu and S. Satija, Stability of thin film glasses of toluene and 
ethylbenzene formed by vapor deposition: an in situ nanocalorimetric study, Phys. Chem. Chem. Phys., 2010, 12, 14693-14698.

3 K. J. Dawson, K. L. Kearns, L. Yu, W. Steffen and M. D. Ediger, Physical vapor deposition as a route to hidden amorphous states, Proc. Natl. Acad. Sci. U. S. A., 2009, 106, 15165-15170.

4 S. S. Dalal, Z. Fakhraai and M. D. Ediger, High-throughput ellipsometric characterization of vapor-deposited indomethacin glasses, J. Phys. Chem. B, 2013, 117, 15415-15425.

5 C. Rodriguez-Tinoco, M. Gonzalez-Silveira, J. Rafols-Ribe, A. F. Lopeandia and J. Rodriguez-Viejo, Transformation kinetics of vapor-deposited thin film organic glasses: the role of stability and molecular packing anisotropy, Phys. Chem. Chem. Phys., 2015, 17, 31195-31201.

6 E. A. A. Pogna, C. Rodríguez-Tinoco, G. Cerullo, C. Ferrante, J. Rodríguez-Viejo and T. Scopigno, Probing equilibrium glass flow up to exapoise viscosities, Proc. Natl. Acad. Sci. U. S. A., 2015, 112, 2331-2336.

7 C. Rodríguez-Tinoco, J. Ràfols-Ribé, M. González-Silveira and J. Rodríguez-Viejo, Relaxation dynamics of glasses along a wide stability and temperature range, Sci. Rep., 2016, 6, 35607.

8 T. Perez-Castaneda, C. Rodriguez-Tinoco, J. Rodriguez-Viejo and M. A. Ramos, Suppression of tunneling two-level systems in ultrastable glasses of indomethacin, Proc. Natl. Acad. Sci. U. S. A., 2014, 111, 11275-11280.

9 C. Rodríguez-Tinoco, M. Gonzalez-Silveira, J. Ràfols-Ribé, G. Garcia and J. Rodríguez-Viejo, Highly stable glasses of celecoxib: Influence on thermo-kinetic properties, microstructure and response towards crystal growth, J. Non-Cryst. Solids, 2015, 407, 256-261.

10 J. Ràfols-Ribé, P.-A. Will, C. Hänisch, M. Gonzalez-Silveira, S. Lenk, J. Rodríguez-Viejo and S. Reineke, High-performance organic light-emitting diodes comprising ultrastable glass layers, Sci. Adv., 2018, 4, eaar8332.

11 Y. Qiu, L. W. Antony, J. J. de Pablo and M. D. Ediger, Photostability Can Be Significantly Modulated by Molecular Packing in Glasses, J. Am. Chem. Soc., 2016, 138, 11282-11289.

12 K. L. Ngai and M. Paluch, Classification of secondary relaxation in glass-formers based on dynamic properties, J. Chem. Phys., 2004, 120, 857-873.

13 H. B. Yu, M. Tylinski, A. Guiseppi-Elie, M. D. Ediger and R. Richert, Suppression of $\beta$ Relaxation in Vapor-Deposited Ultrastable Glasses, Phys. Rev. Lett., 2015, 115, 185501.

14 C. Rodríguez-Tinoco, M. Rams-Baron, K. L. Ngai, K. Jurkiewicz, J. Rodríguez-Viejo and M. Paluch, Secondary relaxation in ultrastable etoricoxib: evidence of correlation with structural relaxation, Phys. Chem. Chem. Phys., 2018, 20, 3939-3945.

15 C. Rodríguez-Tinoco, K. L. Ngai, M. Rams-Baron, J. RodríguezViejo and M. Paluch, Distinguishing different classes of secondary relaxations from vapour deposited ultrastable glasses, Phys. Chem. Chem. Phys., 2018, 20, 21925-21933.

16 M. Rams-Baron, Z. Wojnarowska, K. Grzybowska, M. Dulski, J. Knapik, K. Jurkiewicz, W. Smolka, W. Sawicki, A. Ratuszna and M. Paluch, Toward a Better Understanding of the Physical Stability of Amorphous Anti-Inflammatory Agents: The Roles of Molecular Mobility and Molecular Interaction Patterns, Mol. Pharmaceutics, 2015, 12, 3628-3638.

17 M. Rams-Baron, Z. Wojnarowska, J. Knapik-Kowalczuk, K. Jurkiewicz, A. Burian, M. Wojtyniak, J. Pionteck, M. Jaworska, C. Rodríguez-Tinoco and M. Paluch, The dielectric signature of glass density, Appl. Phys. Lett., 2017, 111, 121902.

18 K. Adrjanowicz, M. Paluch and K. L. Ngai, Determining the structural relaxation times deep in the glassy state of the pharmaceutical Telmisartan, J. Phys.: Condens. Matter, 2010, 22, 125902.

19 K. Adrjanowicz, Z. Wojnarowska, P. Wlodarczyk, K. Kaminski, M. Paluch and J. Mazgalski, Molecular mobility in liquid and glassy states of Telmisartan (TEL) studied by Broadband Dielectric Spectroscopy, Eur. J. Pharm. Sci., 2009, 38, 395-404.

20 M. Mierzwa, S. Pawlus, M. Paluch, E. Kaminska and K. L. Ngai, Correlation between primary and secondary JohariGoldstein relaxations in supercooled liquids: Invariance to changes in thermodynamic conditions, J. Chem. Phys., 2008, 128, 44512.

21 K. Kessairi, S. Capaccioli, D. Prevosto, M. Lucchesi, S. Sharifi and P. A. Rolla, Interdependence of primary and Johari-Goldstein secondary relaxations in glass-forming systems, J. Phys. Chem. B, 2008, 112, 4470-4473.

22 M. Shahin Thayyil, K. L. Ngai, D. Prevosto and S. Capaccioli, Revealing the rich dynamics of glass-forming systems by modification of composition and change of thermodynamic conditions, J. Non-Cryst. Solids, 2015, 407, 98-105.

23 K. L. Ngai, J. Habasaki, D. Prevosto, S. Capaccioli and M. Paluch, Thermodynamic scaling of $\alpha$-relaxation time and viscosity stems from the Johari-Goldstein $\beta$-relaxation or the primitive relaxation of the coupling model, J. Chem. Phys., 2012, 137, 034511.

24 S. Capaccioli, M. Paluch, D. Prevosto, L.-M. Wang and K. L. Ngai, Many-Body Nature of Relaxation Processes in Glass-Forming Systems, J. Phys. Chem. Lett., 2012, 3, 735-743.

25 D. Prevosto, S. Capaccioli, M. Lucchesi, P. A. Rolla and K. L. Ngai, Does the entropy and volume dependence of the structural $\alpha$-relaxation originate from the Johari-Goldstein $\beta$-relaxation?, J. Non-Cryst. Solids, 2009, 355, 705-711.

26 S. Valenti, S. Capaccioli and K. L. Ngai, Contrasting two different interpretations of the dynamics in binary glass forming mixtures, J. Chem. Phys., 2018, 148, 054504.

27 K. L. Ngai and M. Paluch, Corroborative evidences of TV ${ }^{\gamma}$ -scaling of the $\alpha$-relaxation originating from the primitive relaxation/JG $\beta$ relaxation, J. Non-Cryst. Solids, 2017, 478, 1-11.

28 K. L. Ngai, Relaxation and Diffusion in Complex Systems, Springer New York, New York, NY, 2011.

29 K. L. Ngai, M. Paluch and C. Rodríguez-Tinoco, Why is surface diffusion the same in ultrastable, ordinary, aged, and ultrathin molecular glasses?, Phys. Chem. Chem. Phys., 2017, 19, 29905-29912. 
30 Y. Zhang, R. Potter, W. Zhang and Z. Fakhraai, Using tobacco mosaic virus to probe enhanced surface diffusion of molecular glasses, Soft Matter, 2016, 12, 9115-9120.

31 Y. Zhang and Z. Fakhraai, Invariant Fast Diffusion on the Surfaces of Ultrastable and Aged Molecular Glasses, Phys. Rev. Lett., 2017, 118, 066101.

32 Y. Zhang and Z. Fakhraai, Decoupling of surface diffusion and relaxation dynamics of molecular glasses, Proc. Natl. Acad. Sci. U. S. A., 2017, 114, 4915-4919.

33 C. Rodríguez-Tinoco, M. González-Silveira, M. Barrio, P. Lloveras and J. L. Tamarit, J.-L. Garden and J. RodríguezViejo, Ultrastable glasses portray similar behaviour to ordinary glasses at high pressure, Sci. Rep., 2016, 6, 34296.

34 R. Casalini and C. M. Roland, Thermodynamical scaling of the glass transition dynamics, Phys. Rev. E: Stat., Nonlinear, Soft Matter Phys., 2004, 69, 062501.

35 G. Tarjus, D. Kivelson, S. Mossa and C. Alba-Simionesco, Disentangling density and temperature effects in the viscous slowing down of glassforming liquids, J. Chem. Phys., 2004, 120, 6135-6141.

36 C. M. Roland, S. Hensel-Bielowka, M. Paluch and R. Casalini, Supercooled dynamics of glass-forming liquids and polymers under hydrostatic pressure, Rep. Prog. Phys., 2005, 68, 1405-1478.

37 D. Coslovich and C. M. Roland, Thermodynamic Scaling of Diffusion in Supercooled Lennard-Jones Liquids, J. Phys. Chem. B, 2008, 112, 1329-1332.

38 G. Tsolou, V. A. Harmandaris and V. G. Mavrantzas, Atomistic molecular dynamics simulation of the temperature and pressure dependences of local and terminal relaxations in cis-1,4-polybutadiene, J. Chem. Phys., 2006, 124, 084906.

39 D. M. Walters, R. Richert and M. D. Ediger, Thermal stability of vapor-deposited stable glasses of an organic semiconductor, J. Chem. Phys., 2015, 142, 134504.

40 P.-H. Lin, I. Lyubimov, L. Yu, M. D. Ediger and J. J. de Pablo, Molecular modeling of vapor-deposited polymer glasses, J. Chem. Phys., 2014, 140, 204504.

41 S. Singh and J. J. de Pablo, A molecular view of vapor deposited glasses, J. Chem. Phys., 2011, 134, 194903.

42 G. D. Smith and D. Bedrov, Relationship between the $\alpha$ - and $\beta$-relaxation processes in amorphous polymers: Insight from atomistic molecular dynamics simulations of 1,4polybutadiene melts and blends, J. Polym. Sci., Part B: Polym. Phys., 2007, 45, 627-643.

43 S. Kołodziej, S. Pawlus, K. L. Ngai and M. Paluch, Verifying the Approximate Coinvariance of the $\alpha$ and Johari-Goldstein $\beta$ Relaxation Times to Variations of Pressure and Temperature in Polyisoprene, Macromolecules, 2018, 51, 4435-4443.

44 K. L. Ngai, Relation between some secondary relaxations and the $\alpha$ relaxations in glass-forming materials according to the coupling model, J. Chem. Phys., 1998, 109, 6982-6994.

45 K. L. Ngai and K. Y. Tsang, Similarity of relaxation in supercooled liquids and interacting arrays of oscillators, Phys. Rev. E: Stat. Phys., Plasmas, Fluids, Relat. Interdiscip. Top., 1999, 60, 4511-4517.

46 D. Bedrov and G. D. Smith, Secondary Johari-Goldstein relaxation in linear polymer melts represented by a simple bead-necklace model, J. Non-Cryst. Solids, 2011, 357, 258-263.

47 K. L. Ngai, R. Casalini, S. Capaccioli, M. Paluch and C. M. Roland, Do theories of the glass transition, in which the structural relaxation time does not define the dispersion of the structural relaxation, need revision?, J. Phys. Chem. B, 2005, 109, 17356-17360.

48 K. L. Ngai, R. Casalini, S. Capaccioli, M. Paluch and C. M. Roland, Fractals, Diffusion, and Relaxation in Disordered Complex Systems, John Wiley \& Sons, Inc., 2006, pp. 497-593.

49 K. Grzybowska, M. Paluch, A. Grzybowski, Z. Wojnarowska, L. Hawelek, K. Kolodziejczyk and K. L. Ngai, Molecular Dynamics and Physical Stability of Amorphous AntiInflammatory Drug: Celecoxib, J. Phys. Chem. B, 2010, 114, 12792-12801.

50 R. Casalini and C. M. Roland, Density Scaling of the Structural and Johari-Goldstein Secondary Relaxations in Poly(methyl methacrylate), Macromolecules, 2013, 46, 6364-6368.

51 T. C. Ransom, D. Fragiadakis and C. M. Roland, The $\alpha$ and Johari-Goldstein Relaxations in 1,4-Polybutadiene: Breakdown of Isochronal Superpositioning, Macromolecules, 2018, 51, 4694-4698. 